\title{
Coronavirus 19 (COVID-19) infection and vaccination in patients with Shwachman-Diamond syndrome
}

\author{
Thomas Galletta $^{1}$, Sara Loveless ${ }^{1}$, Maggie Malsch $^{2}$, Akiko Shimamura $^{3}$, and Kasiani Myers ${ }^{1}$ \\ ${ }^{1}$ Cincinnati Children's Hospital Medical Center \\ ${ }^{2}$ Boston Children's Hospital \\ ${ }^{3}$ Dana-Farber/Boston Children's Cancer and Blood Disorders Center
}

December 31, 2021

\begin{abstract}
Because they can experience neutropenia due to bone marrow failure, patients with Shwachman-Diamond syndrome (SDS) carry increased risk for serious infections compared to the general population; however, it has been unknown whether this predisposes them to COVID-19 infection or more significant complications. We compiled results from a survey distributed to participants in the Shwachman-Diamond Syndrome Registry between May and June 2021. In this report we describe the characteristics and outcomes of patients with SDS who had COVID-19. Patients reported a short clinical course without significant complications or severe cytopenias. Additionally, COVID-19 vaccines were well tolerated with only minor side effects.
\end{abstract}

\section{Introduction:}

The Coronavirus 19 (COVID-19) pandemic has had significant implications for patients with chronic medical conditions, particularly those who are immunocompromised due to malignancy or bone marrow failure (BMF). Shwachman-Diamond syndrome (SDS) is an inherited BMF disorder characterized by cytopenias, particularly neutropenia, exocrine pancreatic dysfunction, and predisposition to myeloid malignancies. Its manifestations are variable and can affect many body systems, including the skeletal, cardiac, endocrine, nervous, hepatic, and immune systems. In most cases SDS is due to recessive mutations in the ShwachmanBodian-Diamond Syndrome ( $S B D S$ ) gene located on chromosome 7q11. Because patients with SDS can experience neutropenia due to BMF, they carry a higher risk for serious infections compared to the general population; however, it has been unknown whether this predisposes them to developing COVID-19 or experiencing greater complications related to COVID-19.

\section{Methods:}

A survey was distributed to patients participating in the Shwachman-Diamond Syndrome Registry, and responses were anonymous. Results were compiled between May and June 2021. In this cohort study we report the baseline characteristics and clinical outcomes of these ten patients with SDS who had a COVID-19 infection.

\section{Results:}

Of the 73 survey respondents, 10 patients experienced COVID-19 infection (13.7\%) [Table 1]. Seven of these ten patients $(70 \%)$ reported germline SBDS mutations. The median age of the COVID-19 positive patients at time was diagnosis was 16.5 years (range 1-37 years). Of the three patients who both developed COVID-19 infection and received a COVID-19 vaccine, all of them received the vaccine following COVID19 infection, two to six months following infection (median 5 months). None of them experienced serious vaccine-related adverse events. All vaccinated patients received two mRNA vaccine doses. All patients in the 
SDS Registry who developed COVID-19 were symptomatic of infection with the most common symptoms being respiratory, such as congestion or cough (70\%), or fever $(60 \%)$. The median duration of symptoms was 4.5 days (range 2-100 days; excluding an outlier of 100 days, range 4-14 days). Only one of ten patients required hospitalization, and this patient was one year-old (PT-6). She was hospitalized two days after symptom onset and was hospitalized for four days. She did not require supplemental oxygen, noninvasive positive-pressure ventilation, or intubation and mechanical ventilation. She did not require admission to the intensive care unit.

Of the survey respondents who developed COVID-19 infection, none of them reported having a pre-existing condition other than SDS. Pre-existing conditions listed in the survey included hypertension, diabetes mellitus, asthma, and routine need for immune globulin (IVIG). Respondents were able to report other conditions that were not listed. No patients who developed COVID-19 infection reported a history of tobacco use or vaping. Six of them (60\%) reported regularly taking pancreatic enzymes, and two of them (20\%) reported regularly administering granulocyte colony-stimulating factor (G-CSF) prior to COVID infection (PT-8 and PT-9). One patient reported a history of a hematopoietic stem cell transplant over 15 years ago (PT-4).

Survey respondents were asked if they received any of the following COVID-19 infection treatments: remdesivir, hydroxychloroquine/chloroquine, azithromycin, anakinra, tocilizumab, corticosteroids, immune globulin, or the investigational COVID-19 monoclonal antibody, bamlanivimab. One patient (PT-10) received remdesivir, and one patient (PT-4) received systemic corticosteroids. One patient (PT-5) developed "COVID toes" - swelling, discoloration, and/or pain of the toes associated with COVID-19 - and was treated with aspirin and topical corticosteroids. She also reported the longest duration of symptoms out of all respondents, 100 days. No patients required pRBC or platelet transfusion during COVID-19 infection, or new administration of G-CSF. No patients developed a venous thromboembolism, multisystem inflammatory syndrome in adults (MIS-A), or children (MIS-C).

In the cohort of SDS registry patients described, most reported having a short duration of symptoms that did not require hospitalization or result in serious virus-related complications. Although the Centers for Disease Control and Prevention (CDC) recommends receiving the COVID-19 vaccine even after developing COVID-19 infection, only 30\% of patients with SDS and COVID-19 infection subsequently received the vaccine; three of whom were ineligible to receive the vaccine at the time of survey due to age less than 12. Of the 63 survey respondents who had not developed COVID-19 infection, 18 (28.6\%) of them received at least one dose of the COVID-19 vaccine; 15 of whom (83.3\%) received two doses. Of the 18 respondents who received at least one COVID-19 vaccine dose, arm pain was the most common side effect (66.7\%), followed by fatigue $(50 \%)$, fever and/or chills $(38.9 \%)$, and muscle aches $(33.3 \%)$. Other side effects listed in the survey were rash and anaphylaxis; no respondents reported experiencing these symptoms. Five of 18 recipients $(27.8 \%)$ reported experiencing no side effects. Thirteen $(72.2 \%)$ of 18 vaccinated respondents received the Pfizer vaccine while the remainder received the Moderna vaccine.

\section{Discussion:}

In this report we describe the largest cohort to date of patients with SDS and COVID-19 infection. Most patients reported a short clinical course with few requiring COVID-19 directed therapy, and only one requiring hospitalization; none experienced significant complications or severe cytopenias. However, these results cannot be generalized to patients with more severe comorbidities. COVID-19 vaccines were well tolerated with only minor expected side effects, suggesting that vaccination should be encouraged according to CDC guidelines during this ongoing global pandemic.

Conflict of Interest: The authors would like to highlight a potential conflict of interest as Drs. Akiko Shimamura and Kasiani Myers work closely with Dr. Peter Newburger on the Severe Congenital Neutropenia International Registry.

Acknowledgments: The authors gratefully acknowledge all patients and families participating in the SDS Registry as well as physician colleagues who referred patients to the Registry. 


\section{References: None}

Legends: None

\section{Hosted file}

COVID SDS Figure PBC 12.28.21.docx available at https://authorea.com/users/453372/articles/ 551221-coronavirus-19-covid-19-infection-and-vaccination-in-patients-with-shwachmandiamond-syndrome 